\title{
Expression analysis of MIR182 and its associated target genes in advanced ovarian carcinoma
}

\author{
Brian D McMillen ${ }^{1}$, Margarita M Aponte ${ }^{2}$, Zhaojian Liu ${ }^{1}$, Irene B Helenowski ${ }^{3}$, \\ Denise M Scholtens ${ }^{3}$, Barbara M Buttin ${ }^{2,4}$ and Jian-Jun Wei ${ }^{1,4}$ \\ ${ }^{1}$ Department of Pathology, Northwestern University, Feinberg Medical School, Chicago, IL, USA; ${ }^{2}$ Department \\ of Obstetrics and Gynecology, Northwestern University, Feinberg Medical School, Chicago, IL, USA; \\ ${ }^{3}$ Department of Preventive Medicine, Northwestern University, Feinberg Medical School, Chicago, IL, USA \\ and ${ }^{4}$ Robert H. Lurie Comprehensive Cancer Center, Northwestern University, Feinberg Medical School,
} Chicago, IL, USA

\begin{abstract}
BRCA1/BRCA2 mutations are common and the hallmarks of high-grade serous ovarian carcinoma. We found that MIR182, a negative BRCA1 regulator, is significantly overexpressed in high-grade serous ovarian carcinoma. To examine whether overexpression of MIR182 and its target genes, including BRCA1, HMGA2 (high-mobility group A2), FOXO3 and MTSS1, are associated with high-grade serous ovarian carcinoma tumor types and clinical outcome, we studied MIR182 by in situ hybridization and its target gene expression by immunohistochemistry in 117 cases of advanced ovarian cancer. We found that high-grade serous ovarian carcinoma had significantly higher MIR182 $(P=0.0003)$ and HMGA2 $(P=0.04)$ expression, and significantly lower BRCA1 $(P<0.0001)$ and FOXO3 $(P<0.001)$ expression than normal controls. MIR182 is significantly correlated with MTSS1 expression $(r=0.31 ; P<0.001)$, whereas other target genes did not show a significant correlation with MIR182, indicating a complicated regulatory mechanisms of these genes in high-grade serous ovarian carcinoma. Among the examined MIR182 target genes, only HMGA2 was significantly associated with serous type carcinomas $(P<0.01)$, ascites $(P<0.01)$ and high death rate $(P=0.02)$. FOXO3 expression was associated with lower-stage disease $(P=0.04)$ and solid growth pattern $(P=0.03)$. MIR182 expression is significantly higher in high-grade serous ovarian carcinoma than in fallopian tubes.
\end{abstract}

Modern Pathology (2012) 25, 1644-1653; doi:10.1038/modpathol.2012.118; published online 13 July 2012

Keywords: miR-182; ovarian cancer; BRCA1; HMGA2; MTSS1

High-grade serous ovarian carcinoma is an aggressive and deadly form of ovarian cancer, yet its pathogenesis is poorly understood. Despite significant efforts of clinical researchers, the survival rate of women with high-grade serous ovarian carcinoma has not changed in the past 50 years. ${ }^{1}$ These tumors are often high-grade and aggressive at presentation, with a poor prognosis. Recent recognition of the existence of high-grade serous ovarian carcinoma precursor lesions, serous tubal intraepithelial

Correspondence: Dr J-J Wei, MD, Department of Pathology, Northwestern University, SOM, Feinberg 7-334, 251 East Huron Street, Chicago, IL 60611, USA.

E-mail: jianjun-wei@northwestern.edu

Received 25 March 2012; revised 3 May 2012; accepted 14 May 2012; published online 13 July 2012 carcinoma, ${ }^{2,3}$ in the distal (fimbriated) ends of the fallopian tubes has renewed hope that we will be able to identify early tumorigenic events leading to high-grade serous ovarian carcinoma and reveal new opportunities for early detection and treatment that may decrease the mortality rate among women with this cancer.

BRCA1 and BRCA2 mutations are a hallmark of high-grade serous ovarian carcinoma tumorigenesis. ${ }^{4}$ Women with germline BRCA1/2 mutations have a $30-70 \%$ chance of developing high-grade serous ovarian carcinoma by age $70 .^{5}$ Germline $B R C A$ mutations, ${ }^{6}$ somatic mutations and epigenetic inactivation of $B R C A 1 / 2$ (via methylation) can be found in nearly $30 \%$ of high-grade serous ovarian carcinoma cases. ${ }^{7}$ BRCA1 has a broader role upstream of $B R C A 2$, participating in various cellular 
processes in response to DNA damage repair ${ }^{8}$ and transcriptional regulation. ${ }^{9}$ If inactivation of $B R C A 1 / 2$ is critical in high-grade serous ovarian carcinoma cases, other regulation mechanism for BRCA1/2 expression may exist. In their study of cell response to DNA damage, Moskwa et $a l^{10}$ found that MIR182 can specifically repress $B R C A 1$ expression, suggesting a role for microRNA in the regulation of BRCA1 expression. In a recent study of miRNA profiling analysis in normal fallopian tube and serous tubal intraepithelial carcinoma, we found that MIR182 is one of a few miRNAs with significant overexpression in serous tubal intraepithelial carcinoma. ${ }^{11}$

MIR182 is an oncogene (onco-miR) and its oncogenic properties are characterized by its negative regulation of several tumor suppressor genes including BRCA1, ${ }^{10}$ FOXO1, ${ }^{12,13}$ FOXO3 and MIF. ${ }^{14}$ We recently found that $H M G A 2$ (high-mobility group A2) and MTSS1 are the direct and indirect target genes of MIR182. ${ }^{11}$ To better understand the role of MIR182 overexpression in the tumorigenesis of high-grade serous ovarian carcinoma, it is important to examine the expression of MIR182 and its target genes in ovarian cancer cases. It is also important to test whether MIR182-mediated target gene dysregulation is associated with different histological subtypes of ovarian cancer and outcome of the disease. In this study, we examined the expression patterns of MIR182 and its five target genes in a large cohort of advanced ovarian cancer patients, and determined if expression levels correlated with survival or prognostic factors.

\section{Materials and methods}

\section{Case Selection}

Archived tumor tissues were collected retrospectively from patients who underwent surgery for ovarian cancer at the Northwestern Memorial Hospital between 2002 and 2007 and that were treated at our institution by reviewing electronic medical records and paper charts. We selected all patients diagnosed with FIGO stage III or IV epithelial ovarian cancer that had undergone initial surgery at the Northwestern University, including 117 cases (100 high-grade serous ovarian carcinoma, 3 low-grade serous ovarian carcinoma 14 nonserous carcinoma) and 30 normal fallopian tubes were collected as normal tissue controls. Patients' clinical biodemography, pathological and clinical outcomes were collected by retrospective chart review and are summarized in Table 1. Approval of the Institutional Review Board from Northwestern University was obtained.

\section{Tissue Preparation, Antibodies and Immunohistochemistry}

All cases were reviewed by two pathologists. Tissue cores were collected from tumor and control sections of each case (normal fallopian tubes for serous
Table 1 Clinical characteristics of 117 ovarian carcinomas

\begin{tabular}{lc}
\hline Parameters & Scale and range \\
\hline Age, years & \\
$\quad$ Range & $34-90$ \\
Mean \pm s.d. & $58.8 \pm 10.7$ \\
Median & \\
Tumor type & 100 \\
Serous ovarian carcinoma-high grade & 3 \\
Serous ovarian carcinoma-low grade & 14 \\
Nonserous ovarian carcinoma & \\
& \\
Tumor stage & 0 \\
I & 0 \\
II & 3 \\
IIIA & 5 \\
IIIB & 91 \\
IIIC & 18 \\
IV & \\
Lymph node metastases & 13 \\
None & 36 \\
Pelvic & 15 \\
Periaortic & \\
Both & \\
Follow-up (14-82 months) & 24 \\
Alive with disease & 71 \\
Death & 22 \\
Disease free & \\
\hline
\end{tabular}

carcinoma) for tissue microarray and represented in duplicate. Antibodies used for this study included BRCA1 (Dako, Carpinteria, CA, USA; Calbiochem (EMD), Darmstadt, Germany; Abcam, Cambridge, MA, USA), HMGA2 (BioChem, CA, USA), MTSS1 (Neomarkers, Fremont, CA, USA) and FOXO3a (Dako). To obtain better immunohistochemical results from BRCA1, we examined BRCA1 expression by immunohistochemistry from three different sources of anti-BRCA1 (Dako, EMD and Abcam). We found that all antibodies provided variable results for immunointensity, but no statistical significance among antibodies was observed (data not shown). As anti-BRCA1 antibody (MS110) from EMD provided clear and constant results, and has been used in several different studies (see Results and Discussion), we used this antibody for the current study.

Tissue microarrays were sectioned $4 \mu \mathrm{m}$ in thickness. After deparaffinization and antigen retrieval, all immunohistochemical staining was performed on a Ventana Nexus automated system (Tucson, AZ, USA). In brief, endogenous peroxidase activity was blocked with $3 \%$ hydrogen peroxide. Primary antibodies were detected using standard biotinylated anti-mouse or anti-rabbit secondary antibodies.

\section{MIR182 microRNA In Situ Hybridization}

The hybridization system and probes of miRCURY LNA, including MIR182 and U6, were purchased from Exiqon (Vedbaek, Denmark). The detailed 
procedure for in situ hybridization was followed as per the manufacturer's protocol. ${ }^{15}$ In brief, $4-\mu \mathrm{m}$ tissue microarray slides were prepared. Following deparaffinization and deproteinization, the slides were prehybridized with $1 \mathrm{X}$ hybridization buffer without probe. The hybridization was carried out overnight in a $1 \mathrm{X}$ hybridization buffer $(30-70 \mu \mathrm{l})$ with predenatured miRCURY LNA, MIR182 U6 probes. After washing, the slides were blocked and incubated with AP-conjugated anti-DIG Fab fragments (1:1500, Roche, Indianapolis, IN, USA) and visualized for color detection.

\section{Semiquantitative Scores of Immunointensity and Intensity for microRNA In Situ Hybridization}

One-score system for immunointensity and microRNA intensity was used for the markers HMGA2, BRCA1, FOXO3, MTSS1 and microRNA MIR182. The semiquantitation for intensity was scored on a scale of $0-3$, 0: negative, 1: weak, 2: moderate and 3: strong. As tissue cores for all cases were duplicated, the scales for each case and each marker were given by average scores.

\section{Statistical Analysis}

Gene expression levels for the entire patient population as well as separated by cases and controls, and by histology and clinical groups were summarized by medians and ranges. Differences between cases and controls, and among histology and clinical groups were assessed using the Wilcoxon rank-sum tests. To determine associations of clinical parameters (optimal bulking, chemoresistance and survival) with gene expression, expression levels were dichotomized according to levels $\leq 1.5$ or $>1.5$. To evaluate the association of pathological findings with the gene expression, we selected the following histological features for analysis, including tumor type (serous and nonserous), grade (low-grade and high-grade), architecture (glandular, papillary, micropapillary and solid), tumor necrosis $(<10 \%$ and $>10 \%)$ and tumor-infiltrating lymphocyte (absent and present). The associations were evaluated according to Fisher's exact test. The overall survival curves were calculated for the expression groups using the KaplanMeier method with hazard ratios estimated according to the Cox proportional hazards models. Associations among the gene expression levels were assessed via Spearman's correlation. $P$-values $<0.05$ were considered statistically significant.

\section{Results}

\section{Patients' Clinical and Pathological Information and Case Selection}

Cases included in the study were stage III and IV ovarian carcinomas with well-documented histological and clinical information, including tumor types, grade, stage, treatment and follow-up (up to 10 years). The clinical information of these 117 cases is summarized in Table 1. Patients' age ranged from 34 to 90 years (mean age 59). About 85\% (100/ 117) of the cases were high-grade serous carcinoma, $2.5 \%(3 / 117)$ were low-grade serous carcinoma and $12 \%(14 / 117)$ were nonserous carcinomas (including endometrioid, clear cell and mucinous carcinomas). All patients had FIGO stage III and IV disease (stage III $=85 \%$, stage IV $=15 \%$ ) and all underwent surgical debulking. Nearly 60\% (70/117) patients had periaortic and/or pelvic lymph node metastasis. About $60 \%$ of patients died of disease, $20 \%$ were alive with disease at the time of follow-up and another $20 \%$ were disease free during the study period (Table 1). To better estimate the tested gene expression in these 117 cases, we prepared tissue microarrays of 1-mm cores in duplicates, and all cores were randomly distributed throughout tissue microarray blocks. We randomly selected 30 matched fallopian tubes as normal controls and they were also arrayed randomly into the tissue microarray blocks.

\section{Differential Expression of MIR182 and its Target Genes Between Normal (Fallopian Tube) Controls and Ovarian Cancer Cases}

To test whether MIR182 and its target gene expression are associated with ovarian cancer, we examined expression of MIR182 and four well-characterized target genes (BRCA1, FOXO3a, HMGA2 and MTSS1) in 117 advanced ovarian carcinomas and normal fallopian tube controls. We found that MIR182 expression was significantly higher in ovarian cancer than in fallopian tube epithelia $(P<0.0003$; Table 2 , Figure 1). The mean and median of MIR182 expression were slightly higher in high-grade serous ovarian carcinoma than in nonserous carcinoma, but this difference was not statistically significant $(P>0.05)$.

The selected MIR182 target gene expressions were evaluated by semiquantitative scoring of immunoreactivity. As shown in Table 2, BRCA1 and FOXO3a had significantly lower immunointensity in ovarian cancer than in fallopian tubes $(P<0.001)$. In contrast, immunoreactivity for HMGA2 was significantly higher in tumors than in controls $(P=0.04)$. MTSS1 had low expression in both carcinoma and control tissue and differences in expression between tumors and controls were insignificant $(P>0.05)$. The findings suggested that MIR182 and its associated target genes were differentially expressed between ovarian carcinomas and control tissues (Table 2, Figure 1).

\section{MIR182 and its Target Gene Expression in Association} with Tumor Types and Pathological Features

To investigate whether expression of MIR182 and its target genes is associated with tumor histological 
Table 2 Summary statistics for MIR182 and its target gene expression in normal fallopian tube and ovarian cancer and $P$-values obtained from the Wilcoxon rank-sum test used to compare fallopian tube and ovarian cancer

\begin{tabular}{|c|c|c|c|c|c|}
\hline \multirow[t]{2}{*}{ Markers } & \multicolumn{2}{|c|}{$\begin{array}{c}\text { Fallopian } \\
\text { tube }(\mathrm{N}=30)\end{array}$} & \multicolumn{2}{|c|}{$\begin{array}{c}\text { Ovarian } \\
\text { cancer }(\mathrm{N}=117)\end{array}$} & \multirow[t]{2}{*}{ P-values } \\
\hline & Median & $\begin{array}{l}\text { Minimum- } \\
\text { maximum }\end{array}$ & Median & $\begin{array}{l}\text { Minimum- } \\
\text { maximum }\end{array}$ & \\
\hline MIR182 & 1.00 & $1.00-3.00$ & 2.00 & $0.50-3.00$ & 0.0003 \\
\hline$B R C A 1$ & 2.00 & $1.00-3.00$ & 1.00 & $0.00-3.00$ & $<\mathbf{0 . 0 0 0 1}$ \\
\hline FОХОЗ & 3.00 & $0.00-3.00$ & 1.50 & $0.00-3.00$ & $<0.001$ \\
\hline MTSS1 & 1.00 & $0.00-2.00$ & 1.00 & $0.00-2.50$ & 0.21 \\
\hline$H M G A 2$ & 1.00 & $0.00-3.00$ & 1.50 & $0.00-3.00$ & 0.04 \\
\hline
\end{tabular}

The bold $P$-values represent the $P<0.05$, meaning statistical significance. features, we selected the following pathomorphological parameters for the analyses: serous and nonserous, solid and non-solid (glandular; papillary and micropapillary) growth patterns; tumor-infiltrating lymphocytes; necrosis, lymph node metastasis, ascites and tumor stages. We found solid growth pattern had significantly lower BRCA1 and FOXO3a expression $(P<0.001$ and 0.02 , respectively $)$. HMGA2 overexpression was significantly associated with ascites and serous carcinoma $(P=0.009$ and 0.01 , respectively). The latter finding was consistent with our previous study. ${ }^{16}$ MIR182 overexpression and FOXO3a downregulation were significantly associated with advanced stage IV carcinoma $(P<0.02$ and 0.07 , respectively). No significant asso-

\section{Fallopian Tube}
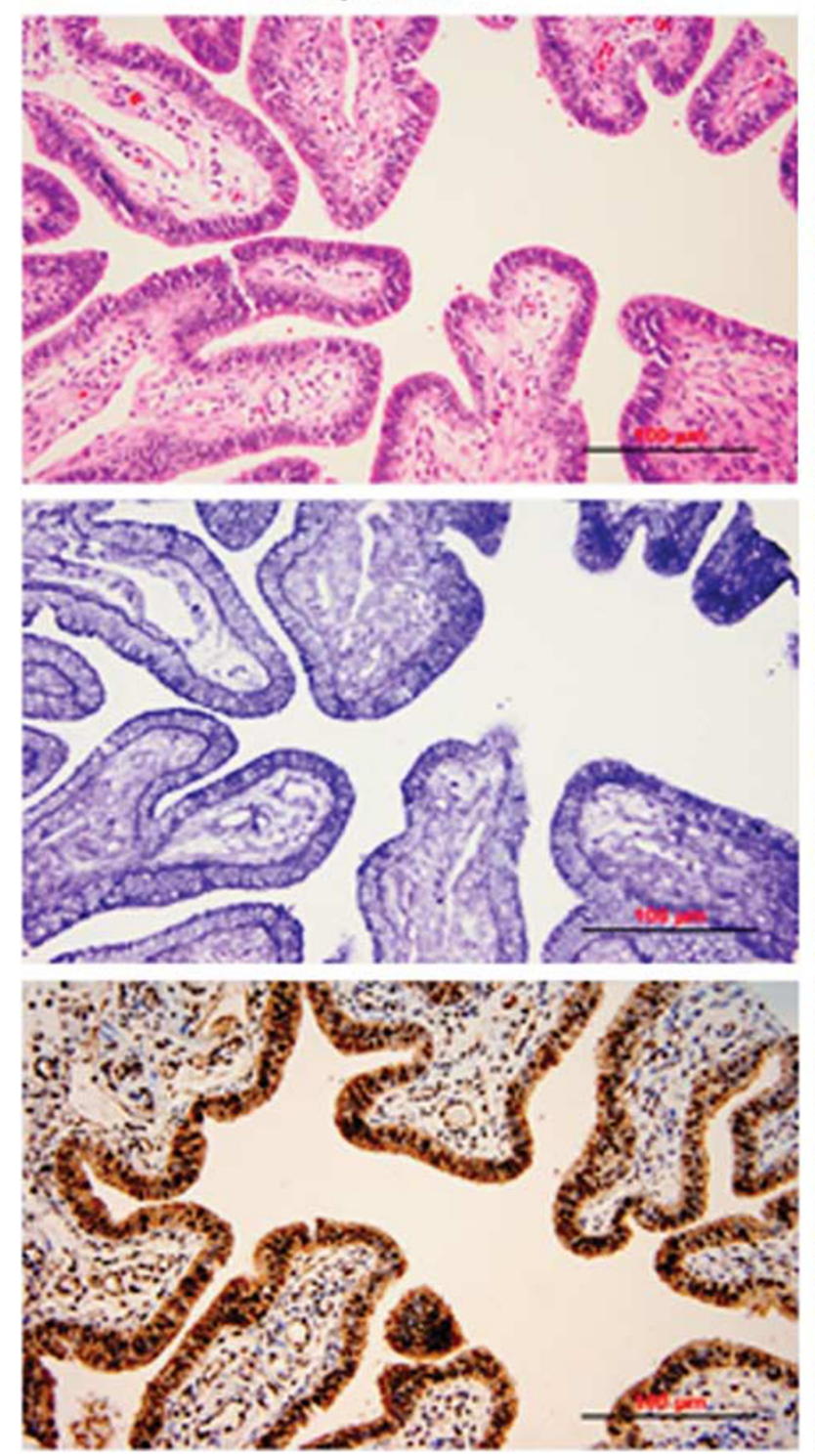

High grade carcinoma
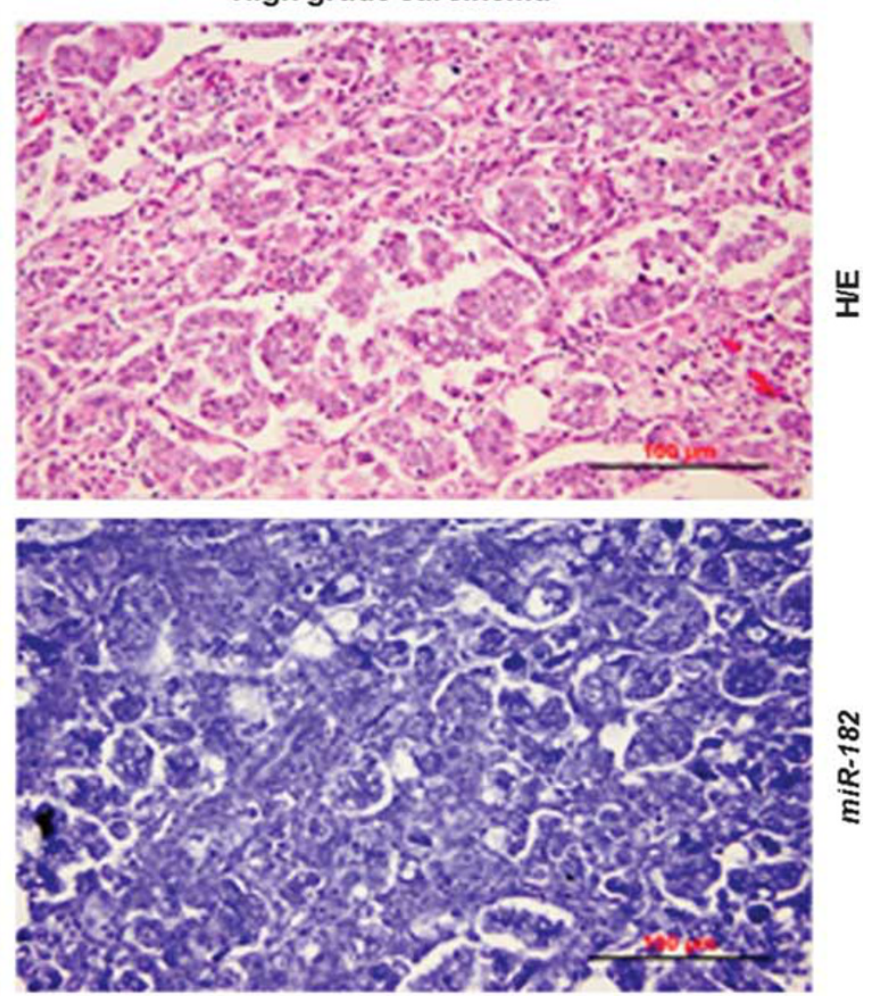

\section{$\stackrel{\infty}{\frac{\infty}{\dot{~}}}$}

Figure 1 Photomicrographs illustrate examples of immunoreactivity for HMGA2, BRCA1, FOXO3, MTSS1 and in situ hybridization of MIR182 in high-grade serous ovarian carcinoma and normal control of fallopian tube. Hematoxylin and eosin (H/E) and immunohistochemical stains for the selected markers were performed in serial sections of tissue core with high magnification $(\times 20)$. 
Fallopian Tube
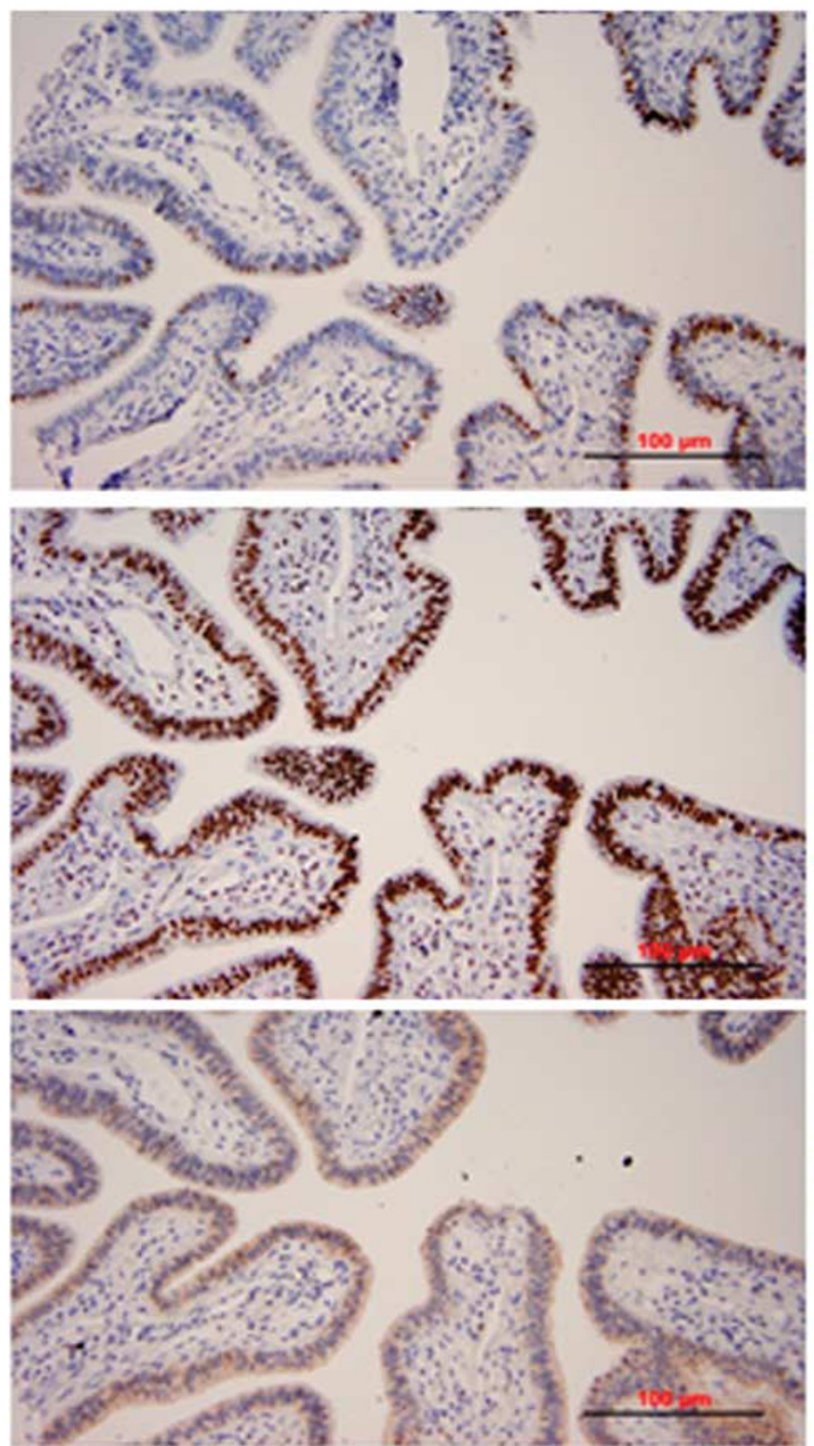

Figure 1 Continued.

ciation of tumor-infiltrating lymphocytes with any marker was found (Table 3).

\section{MIR182 and its Target Gene Expression in Association with Clinical Features}

We selected three clinically most relevant parameters for the analysis: optimal debulking status post surgery, chemoresistance and progression-free survival. To better define the cutoff for the scores of each markers, we compared the differences in the scales of: (1) positive (1-3)/negative (0); (2) negative + weakly positive $(0+1)$ /positive $(2-3)$; or (3) low positive $(<1.5)$ /high positive (1.5). No significant association was found between MIR182 or the other selected protein expression and optimal debulking status post surgery or chemoresistance.
High grade carcinoma
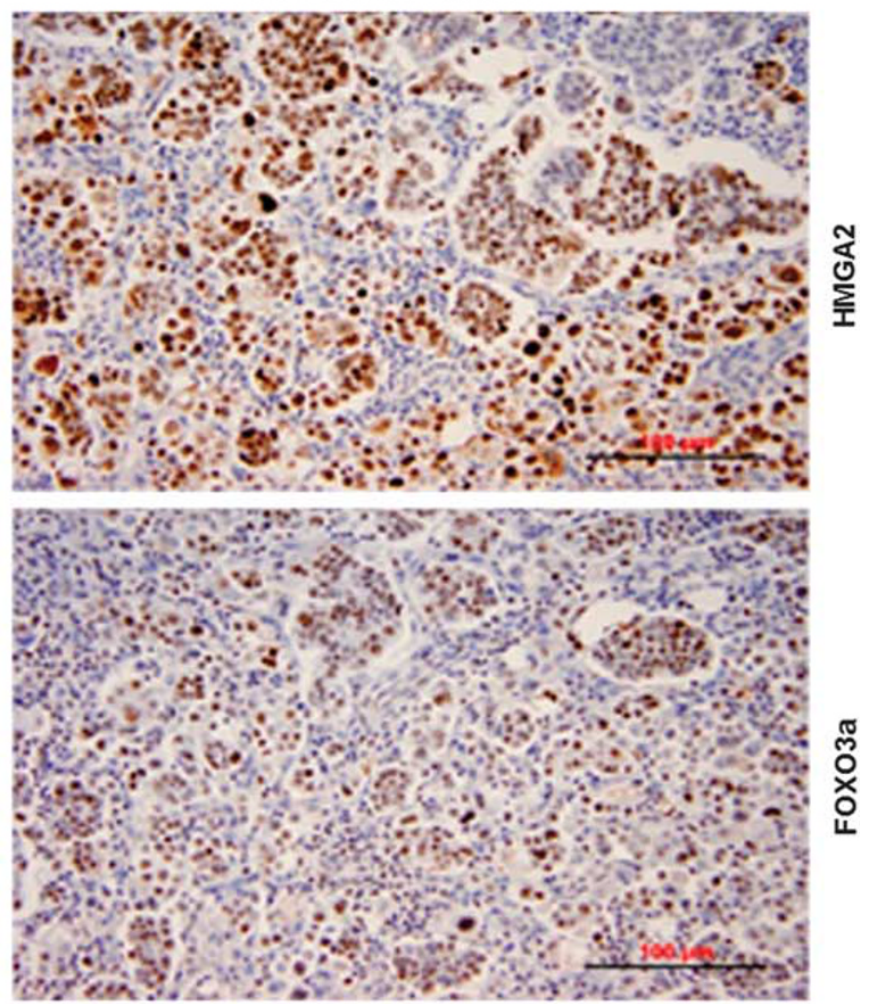

్ㅠ

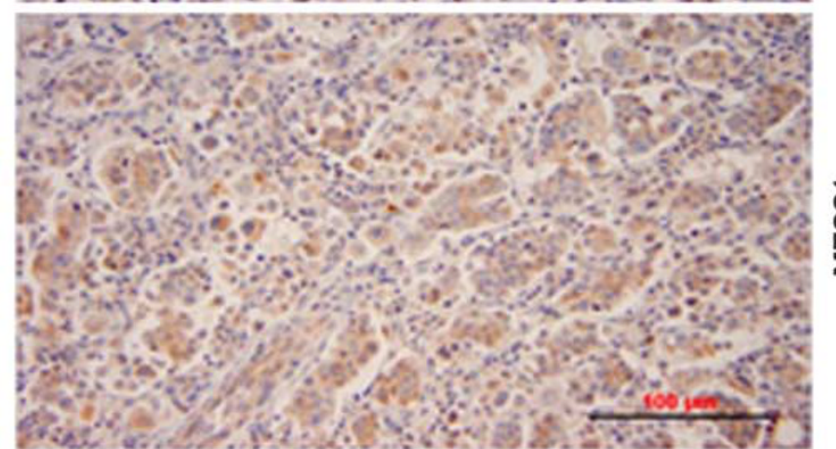

$\stackrel{\bar{s}}{\stackrel{5}{\Sigma}}$
For the dichotomized expression scores $(\leq 1.5$ or $>1.5$ ), there was no significant association between BRCA1 protein expression and overall survival or MIR182 expression and progression-free survival (for $B R C A 1$, adjusted hazard ratio $=0.89(0.52-1.54)$, $P=0.68$; for $M I R 182$, adjusted hazard ratio $=0.89$ (0.53-1.51), $P=0.68)$. In the 117 patients, there was a significant association between HMGA2 protein expression and progression-free survival (adjusted hazard ratio $=1.79$ (1.09-2.95), $P=0.02$; Figure 2). The results of other markers are summarized in Table 4. In further analyses classifying patients with minimal residual disease into low $(B R C A 1 \leq 2.5)$ and high $(B R C A 1>2.5)$ expression groups patients with low BRCA1 expression had a more favorable outcome (median progression-free survival was 24.7 and 16.6 months in patients with low and high BRCA1, respectively; hazard ratio $=0.56(0.35-0.89), P=0.01)$. 
Table 3 Summary statistics for MIR182 and its target gene expression in different stages, ascites status, tumor types and architecture, and $P$-values obtained from the Wilcoxon rank-sum test used to compare values between groups

\begin{tabular}{|c|c|c|c|c|c|}
\hline \multirow[t]{2}{*}{ Markers } & \multicolumn{2}{|c|}{ Stage III $(\mathrm{N}=94)$} & \multicolumn{2}{|c|}{ Stage IV $(\mathrm{N}=17)$} & \multirow[t]{2}{*}{ P-values } \\
\hline & Median & Minimum-maximum & Median & Minimum-maximum & \\
\hline MIR182 & 2.00 & $0.50-3.00$ & 1.50 & $1.00-2.50$ & 0.02 \\
\hline$B R C A 1$ & 1.00 & $0.00-3.00$ & 1.50 & $0.50-3.00$ & 0.53 \\
\hline FOXO3a & 1.50 & $0.00-3.00$ & 1.00 & $0.00-2.50$ & 0.07 \\
\hline MTSS1 & 1.00 & $0.00-2.50$ & 0.50 & $0.00-2.00$ & 0.27 \\
\hline \multirow[t]{3}{*}{$H M G A 2$} & 1.50 & $0.00-3.00$ & 1.00 & $0.00-3.00$ & 0.85 \\
\hline & \multicolumn{2}{|c|}{ Ascites $(\mathrm{N}=81)$} & \multicolumn{2}{|c|}{ No ascites $(\mathrm{N}=20)$} & \\
\hline & Median & Minimum-maximum & Median & Minimum-maximum & \\
\hline MIR182 & 2.00 & $0.50-3.00$ & 2.00 & $1.00-3.00$ & 0.92 \\
\hline$B R C A 1$ & 1.50 & $0.00-3.00$ & 1.00 & $0.00-3.00$ & 0.43 \\
\hline FOXO3a & 1.50 & $0.00-3.00$ & 1.50 & $0.00-2.00$ & 0.30 \\
\hline MTSS1 & 1.00 & $0.00-2.50$ & 0.75 & $0.00-2.00$ & 0.30 \\
\hline \multirow[t]{3}{*}{$H M G A 2$} & 2.00 & $0.00-3.00$ & 0.50 & $0.00-3.00$ & 0.01 \\
\hline & \multicolumn{2}{|c|}{$H G S O C(\mathrm{~N}=97)$} & \multicolumn{2}{|c|}{$N O N(\mathrm{~N}=14)$} & \\
\hline & Median & Minimum-maximum & Median & Minimum-maximum & \\
\hline MIR182 & 2.00 & $0.50-3.00$ & 2.00 & $1.00-3.00$ & 0.19 \\
\hline$B R C A 1$ & 1.00 & $0.00-3.00$ & 1.25 & $0.00-2.50$ & 0.82 \\
\hline FOXO3a & 1.50 & $0.00-3.00$ & 2.00 & $1.00-3.00$ & 0.24 \\
\hline MTSS1 & 1.00 & $0.00-2.50$ & 0.75 & $0.00-2.00$ & 0.37 \\
\hline \multirow[t]{3}{*}{ HMGA2 } & 1.50 & $0.00-3.00$ & 0.00 & $0.00-0.00$ & 0.01 \\
\hline & \multicolumn{2}{|c|}{ Papillary/glandular $(\mathrm{N}=52)$} & \multicolumn{2}{|c|}{ Solid $(\mathrm{N}=60)$} & \\
\hline & Median & Minimum-maximum & Median & Minimum-maximum & \\
\hline MIR182 & 2.00 & $1.00-3.00$ & 2.00 & $0.50-3.00$ & 0.51 \\
\hline$B R C A 1$ & 1.50 & $0.00-3.00$ & 1.00 & $0.00-3.00$ & 0.001 \\
\hline FOXO3a & 2.00 & $0.00-3.00$ & 1.50 & $0.00-3.00$ & 0.06 \\
\hline MTSS1 & 1.00 & $0.00-2.50$ & 1.00 & $0.00-2.00$ & 0.55 \\
\hline HMGA2 & 1.50 & $0.00-3.00$ & 1.50 & $0.00-3.00$ & 0.25 \\
\hline
\end{tabular}

The bold $P$-values represent the $P<0.05$, meaning statistical significance.

\section{BRCA1 Immunohistochemical Scores in Ovarian Cancer}

Immunohistochemical stain for BRCA1 is not well established. As described in Materials and methods, we have examined several anti-BRCA1 antibodies from different vendors. We could not get reliable results from Abcam's antibody, but we did obtain a comparable result from EMD and Dako antibodies. Figure 3 provides the examples of side-by-side comparison of immunoreactivity for BRCA1 from these two antibodies. By reviewing the literatures, we found that most authors used MS110 for the study of BRCA1 expression in ovarian cancer (Table 5), we therefore used EMD anti-BRCA1 antibody (MS110) for this study. We found that immunoreactivity for BRCA1 was completely negative in $13 \%$ (14/105), weakly positive in $38 \%$ (40/105), moderately positive in $39 \%(41 / 105)$ and strongly positive in only $10 \%(10 / 105)$ of cases

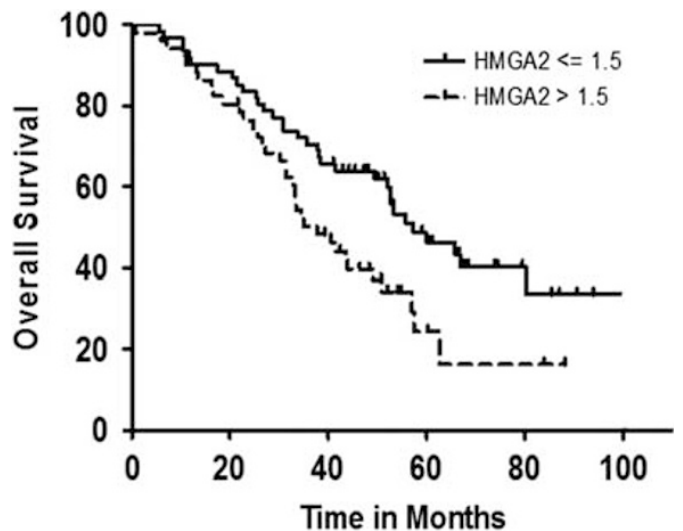

Figure 2 Kaplan-Meier survival curve analysis of HMGA2 in 117 advanced high-grade serous ovarian cancer patients.

(Table 5). Among the 117 ovarian cancer cases, only 6 patients were documented with either BRCA1 or BRCA2 mutations. As not all cases were tested for 
Table 4 MIR182 and its target genes in overall survival

\begin{tabular}{lcccc}
\hline $\begin{array}{l}\text { Prognostic } \\
\text { factors }\end{array}$ & $\begin{array}{c}\text { No. of } \\
\text { cases }\end{array}$ & $\begin{array}{c}\text { No. of } \\
\text { events }\end{array}$ & $\begin{array}{c}\text { Overall survival }^{\mathrm{a}} \\
\text { hazard ratio } \\
(95 \% \text { CI) }\end{array}$ & P-value \\
\hline MIR182 >1.5 & 107 & 64 & $0.89(0.53,1.51)$ & 0.68 \\
BRCA1 >1.5 & 105 & 64 & $0.89(0.52,1.54)$ & 0.68 \\
FOXO3 >1.5 & 108 & 66 & $0.84(0.51,1.37)$ & 0.47 \\
MTSS1 >1.5 & 106 & 64 & $0.51(0.12,2.10)$ & 0.35 \\
HMGA2 >1.5 & 108 & 66 & $1.79(1.09,2.95)$ & $\mathbf{0 . 0 2}$ \\
\hline
\end{tabular}

a The median time and 95\% CI for the whole cohort is: 52.07 (38.14, 57.43) months; the median time and $95 \%$ CI for patients with HMAGA2 score $\leq 1.5$ is: $57.33(49.45,100.17)$ months; and the median time and $95 \%$ CI for patients with HMAGA2 score $>1.5$ is: 37.75 (30.16, 50.83) months.

The bold $P$-values represent the $P<0.05$, meaning statistical significance.

BRCA mutations, comparison of BRCA mutations with immunohistochemistry for BRCA1 could not be further evaluated in this study.

\section{Discussion}

TP53 mutations are common in high-grade serous ovarian carcinoma ${ }^{17}$ and are important in the tumorigenesis of this cancer. However, TP53 mutations are neither sufficient to trigger a sequence of neoplasia nor rate-limiting. ${ }^{4}$ In fact, TP53 mutations are commonly seen in preneoplastic fallopian tube secretory epithelial cells (called a p53 signature) 2,3 $^{2,3}$ and are equally distributed in fallopian tubes of at-risk and non-at-risk populations. ${ }^{18}$ In contrast, BRCA1 and BRCA2 mutations are a hallmark of high-grade serous ovarian carcinoma tumorigenesis. ${ }^{4}$ Based on a recent genome-wide molecular study of ovarian carcinoma from the Cancer Genome Atlas Research Network, 20.25\% ovarian carcinomas had either germ line $(14.56 \%)$ and somatic $(6.01 \%)$ mutations of BRCA1 and BRCA2. ${ }^{19}$ If we combined those ovarian cancer with epigenetic inactivation of BRCA1 and BRCA2 (via methylation), ${ }^{7}$ nearly $30 \%$ of ovarian serous carcinoma may have either defects or altered expression of $B R C A 1 / 2$. However, based on several studies of $B R C A 1$ protein expression analyses (by immunohistochemistry), the absence of or low BRCA1 expression was found in about $41-65 \%$ of ovarian cancer $^{20-22}$ (Table 5), indicating that there are additional as yet undefined mechanisms of BRCA1 inactivation involved in ovarian cancer development. Some of these data were further validated and correlated with BRCA1 mRNA expression. ${ }^{20-22}$

Recent identification of MIR182 repression of $B R C A 1$ expression $^{10}$ provides a new venue for the study of microRNA-mediated BRCA1 gene alteration. In the global profiling analyses of high-grade serous ovarian carcinoma, we $\mathrm{e}^{11}$ and others ${ }^{23}$ found that MIR182 was significantly overexpressed in both early and late stages of this cancer. The finding prompted us to hypothesize that MIR182 may have an important role in the tumorigenesis of high-grade serous ovarian carcinoma; partially through negative regulation of BRCA1 expression and other target genes. In this study, using microRNA in situ hybridization analysis, we found that nearly $70 \%$ of advanced ovarian cancers had upregulation of MIR182 expression in comparison with normal fallopian tubal epithelia (Figure 1, Table 2). This finding suggests that MIR182 overexpression may be responsible for some of the additional cases of low or absent BRCA1 expression in ovarian cancer via BRCA1 downregulation. However, because of the lower sensitivity of semiquantitative immunostaining, no significant inverse correlation between MIR182 and BRCA1 expression was identified in this study.

HMGA2 was one of oncogenes significantly overexpressed in high-grade serous ovarian carcinoma. ${ }^{16,17}$ The major tumorigenic functions of HMGA2 in the pathogenesis of high-grade serous ovarian carcinoma likely include regulation of epithelial mesenchymal transition. ${ }^{24} \mathrm{~A}$ study by Ahmed et $a l^{25}$ shows that BRCA1, ZBRK1 and CtIP form a repression complex that coordinately inhibits $H M G A 2$ expression via a ZBRK1 recognition site in the HMGA2 promoter. We found that MIR182 repression of BRCA1 expression will release a negative regulation and result in HMGA2 overexpression. ${ }^{11,25}$ We propose that MIR182 overexpression in high-grade serous ovarian carcinoma may be in part or completely responsible for HMGA2 overexpression. Although MIR182 and HMGA2 overexpression are found in nearly $70 \%$ of high-grade serous ovarian carcinoma (this study and Mahajan et $a l^{16}$ and Wei et $a 1^{17}$ ), low correlation of expression of these two genes was found $(r=0.23)$ in this study. This could be due to the fact that HMGA2 can be regulated by many microRNAs, such as the wellcharacterized ones in the let-7 family. ${ }^{26,27}$ Nevertheless, we found HMGA2 overexpression is significantly associated with high-grade serous ovarian carcinoma $^{16}$ in this study (Table 4).

FOXO3a is considered a tumor suppressor and is involved in a large series of functions, including cellular proliferation, transformation, differentiation, DNA damage response and longevity. ${ }^{28}$ FOXO3a expression in ovarian cancer is largely unknown. Fei et $a l^{29}$ studied a total of 63 ovarian cancer cases and found that low FOXO3a expression was significantly associated with worse clinical outcome. As a MIR182-specific target gene, ${ }^{14}$ FOX$O 3 a$ expression is of great interest in ovarian cancer. In this study, we did find significant downregulation of FOXO3a in most ovarian cancer cases. The role of $F O X O 3 a$ in ovarian cancer thus deserves further investigation.

MTSS1 is a potential metastasis suppressor gene ${ }^{30}$ and is downregulated in many solid tumors. MTSS1 downregulation enhances the growth, invasion and mobility of breast cancer cells, and is associated with poor prognosis. ${ }^{31}$ We confirmed that MTSS1 is 
a specific target of MIR182 and downregulation of MTSS1 is strongly associated with aggressive invasion of ovarian cancer cells. ${ }^{11}$ This study reveals no significant correlation of MTSS1 expres- sion with pathological and clinical parameters (Table 2).

In summary, this study intends to disclose the expression patterns of MIR182 and its major target
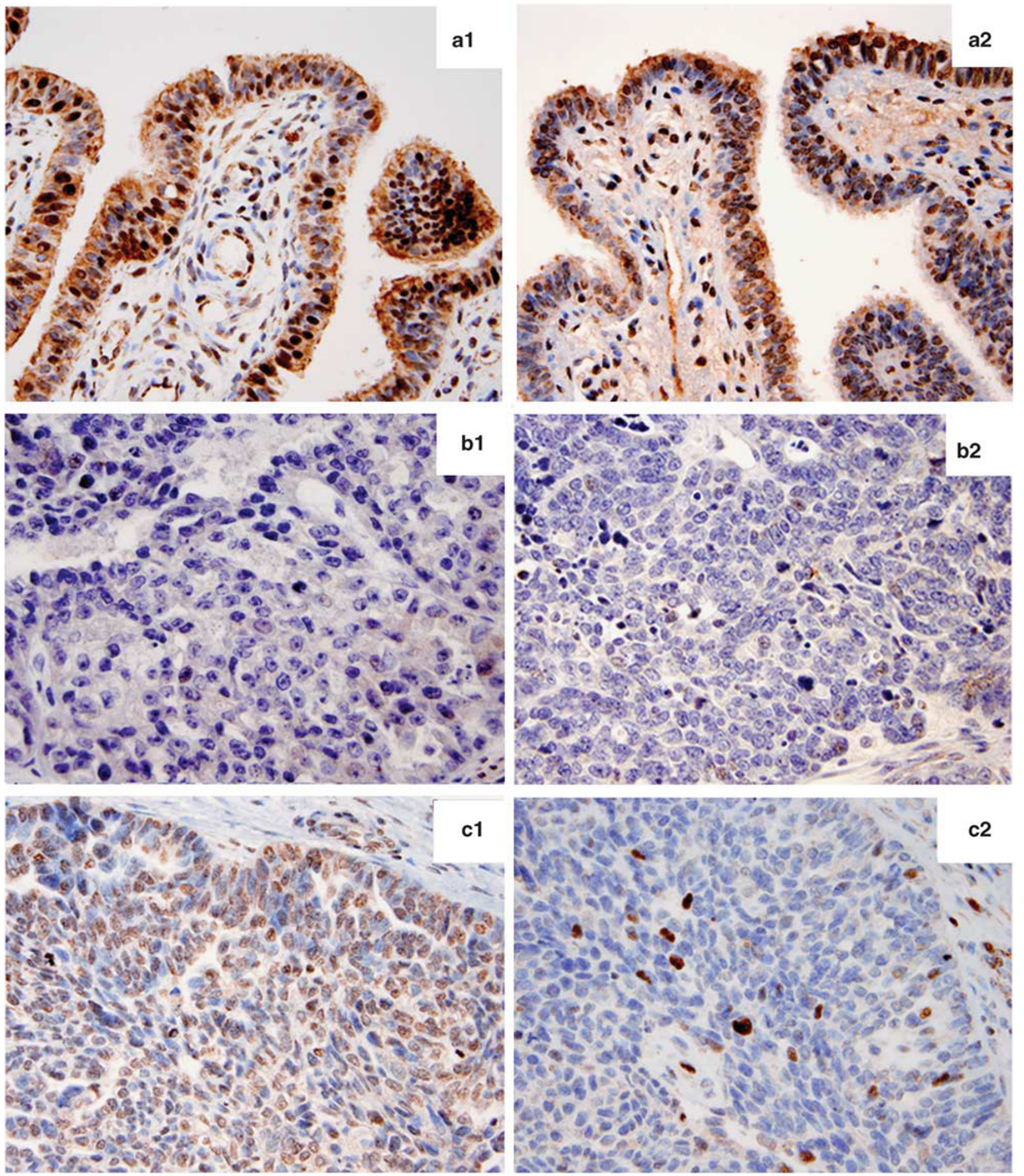

Figure 3 Photomicrographs illustrate examples of immunoreactivity for BRCA1 detected by two different antibodies in normal fallopian tube (a) and high-grade serous ovarian carcinomas (b-e). Immunostain by MS110 from EMD (a1-e1) and GLK from Dako (a2-e2) are shown in side-by-side comparison. Immunointensity for BRCA1 was scored based on MS110 as negative (b), weak (c), moderate (d) and strong (e) expressions. 

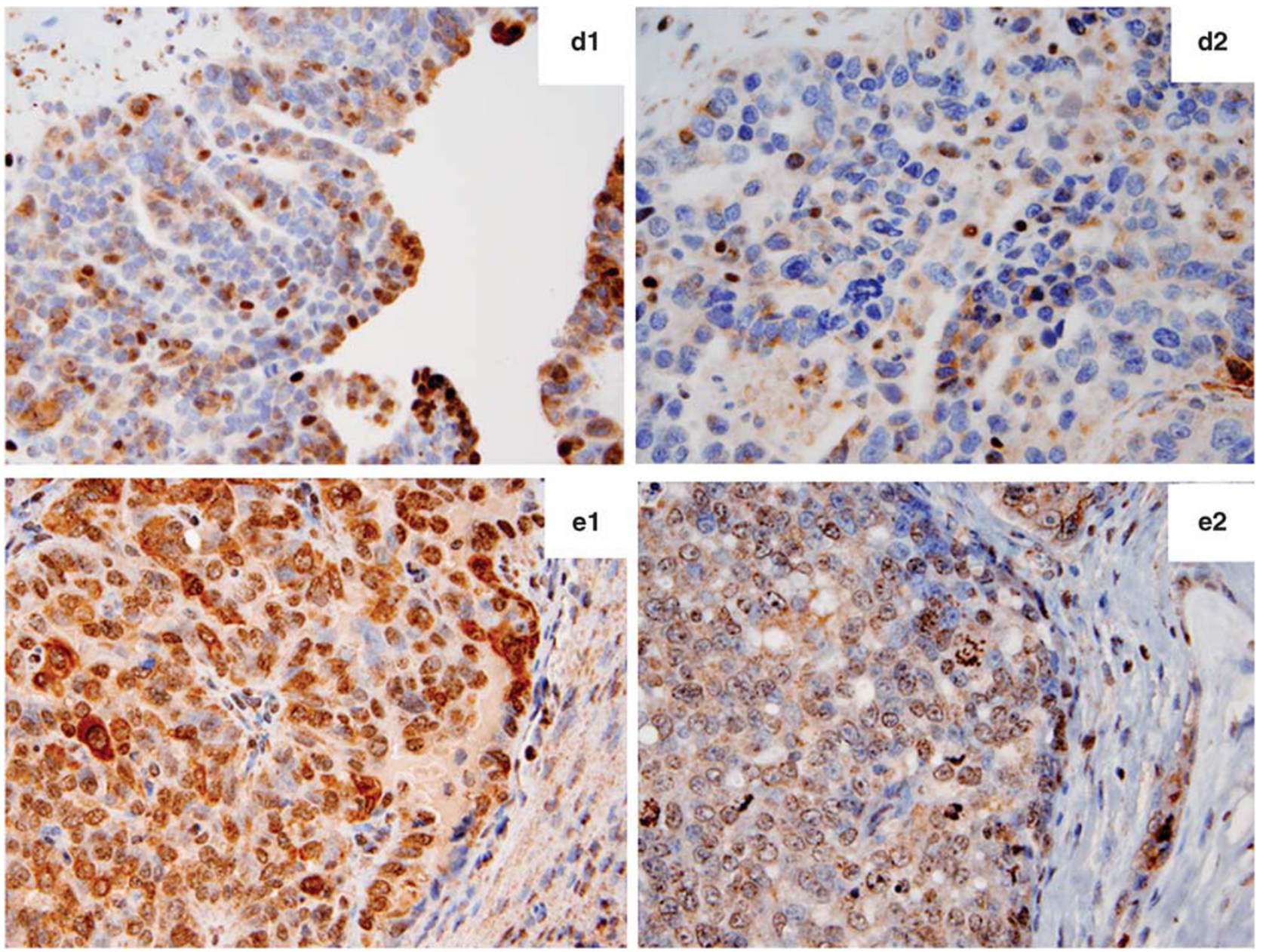

Figure 3 Continued.

Table 5 BRCA1 protein expression analyses scored by immunohistochemistry in this study and studies published recently

\begin{tabular}{|c|c|c|c|c|c|c|}
\hline \multirow[t]{2}{*}{ No. of cases } & \multirow[t]{2}{*}{ BRCA1 antibody } & \multicolumn{4}{|c|}{ Immunoreactivity } & \multirow[t]{2}{*}{ References } \\
\hline & & Negative (\%) & Weak (\%) & Moderate (\%) & Strong (\%) & \\
\hline 292 & MS110 & 41 & & 5 & & Carser et $a l^{21}$ \\
\hline 27 & MS110 & 44 & & 5 & & Radosa et $a l^{20}$ \\
\hline 251 & MS110 & 16 & 49 & 24 & 11 & Weberpals et $a l^{22}$ \\
\hline 117 & MS110 & 13 & 38 & 39 & 10 & McMillen et al (this study) \\
\hline
\end{tabular}

genes in ovarian cancer. We found that at least $50 \%$ of ovarian cancer cases had either absent or low BRCA1 protein expression. These findings are consistent with several similar studies. Among the MIR182 target genes studied, HMGA2 is the only gene that is significantly associated with high-grade and aggressive ovarian cancer in our series. Although we found a significant dysregulation of other selected genes in ovarian cancer, correlation between MIR182 and its target genes has not been established, indicating a complex regulation of these genes in ovarian cancer and lower sensitivity for semiquantitative immunohistochemical scores.

\section{Acknowledgements}

We thank Mrs Bella Shmaltsuyeba for her technical support. This study is supported in part by the Marsha Rivkin Ovarian Cancer Research Award and the Dixon Innovation Translational Research Award. A part of this work was presented in the 101st 
United States and Canadian Academy of Pathology in Vancouver 2012.

\section{Disclosure/conflict of interest}

The authors declare no conflict of interest.

\section{References}

1 Kurman RJ, Shih IeM. The origin and pathogenesis of epithelial ovarian cancer: a proposed unifying theory. Am J Surg Pathol 2010;34:433-443.

2 Medeiros F, Muto MG, Lee Y, et al. The tubal fimbria is a preferred site for early adenocarcinoma in women with familial ovarian cancer syndrome. Am J Surg Pathol 2006;30:230-236.

3 Levanon K, Crum C, Drapkin R. New insights into the pathogenesis of serous ovarian cancer and its clinical impact. J Clin Oncol 2008;26:5284-5293.

4 Bowtell DD. The genesis and evolution of high-grade serous ovarian cancer. Nat Rev Cancer 2010;10: 803-808.

5 Risch HA, McLaughlin JR, Cole DE, et al. Population BRCA1 and BRCA2 mutation frequencies and cancer penetrances: a kin-cohort study in Ontario, Canada. J Natl Cancer Inst 2006;98:1694-1706.

6 Zhang S, Royer R, Li S, et al. Frequencies of BRCA1 and BRCA2 mutations among 1,342 unselected patients with invasive ovarian cancer. Gynecol Oncol 2011;121:353-358.

7 Press JZ, De Luca A, Boyd N, et al. Ovarian carcinomas with genetic and epigenetic BRCA1 loss have distinct molecular abnormalities. BMC Cancer 2008;8:17.

8 Gudmundsdottir K, Ashworth A. The roles of BRCA1 and BRCA2 and associated proteins in the maintenance of genomic stability. Oncogene 2006;25:58645874.

9 Mullan PB, Quinn JE, Harkin DP. The role of BRCA1 in transcriptional regulation and cell cycle control. Oncogene 2006;25:5854-5863.

10 Moskwa P, Buffa FM, Pan Y, et al. miR-182-mediated downregulation of BRCA1 impacts DNA repair and sensitivity to PARP inhibitors. Mol Cell 2011;41: 210-220.

11 Liu Z, Liu J, Segura MF, et al. MiR182 overexpression in tumorigenesis of high-grade ovarian papillary serous carcinoma. J Pathol 2012.

12 Guttilla IK, White BA. Coordinate regulation of FOXO1 by miR-27a, miR-96, and miR-182 in breast cancer cells. J Biol Chem 2009;284:23204-23216.

13 Myatt SS, Wang J, Monteiro LJ, et al. Definition of microRNAs that repress expression of the tumor suppressor gene FOXO1 in endometrial cancer. Cancer Res 2010;70:367-377.

14 Segura MF, Hanniford D, Menendez S, et al. Aberrant miR-182 expression promotes melanoma metastasis by repressing FOXO3 and microphthalmia-associated transcription factor. Proc Natl Acad Sci USA 2009;106: 1814-1819.
15 Kloosterman WP, Wienholds E, Ketting RF, et al. Substrate requirements for let-7 function in the developing zebrafish embryo. Nucleic Acids Res 2004;32: 6284-6291.

16 Mahajan A, Liu Z, Gellert L, et al. HMGA2: a biomarker significantly overexpressed in high-grade ovarian serous carcinoma. Mod Pathol 2010;23:673-681.

17 Wei JJ, Wu J, Luan C, et al. HMGA2: a potential biomarker complement to P53 for detection of earlystage high-grade papillary serous carcinoma in fallopian tubes. Am J Surg Pathol 2010;34:18-26.

18 Lee Y, Miron A, Drapkin R, et al. A candidate precursor to serous carcinoma that originates in the distal fallopian tube. J Pathol 2007;211:26-35.

19 Cancer Genome Atlas Research Network. Integrated genomic analyses of ovarian carcinoma. Nature 2011;474:609-615.

20 Radosa MP, Hafner N, Camara O, et al. Loss of BRCA1 protein expression as indicator of the BRCAness phenotype is associated with favorable overall survival after complete resection of sporadic ovarian cancer. Int J Gynecol Cancer 2011;21:1399-1406.

21 Carser JE, Quinn JE, Michie CO, et al. BRCA1 is both a prognostic and predictive biomarker of response to chemotherapy in sporadic epithelial ovarian cancer. Gynecol Oncol 2011;123:492-498.

22 Weberpals JI, Tu D, Squire JA, et al. Breast cancer 1 (BRCA1) protein expression as a prognostic marker in sporadic epithelial ovarian carcinoma: an NCIC CTG OV.16 correlative study. Ann Oncol 2011;22: 2403-2410.

23 Vaksman O, Stavnes HT, Kaern J, et al. miRNA profiling along tumor progression in ovarian carcinoma. J Cell Mol Med 2010;15:1593-1602.

$24 \mathrm{Wu}$ J, Liu Z, Shao C, et al. HMGA2 overexpressioninduced ovarian surface epithelial transformation is mediated through regulation of EMT genes. Cancer Res 2011;71:349-359.

25 Ahmed KM, Tsai CY, Lee WH. Derepression of HMGA2 via removal of ZBRK1/BRCA1/CtIP complex enhances mammary tumorigenesis. J Biol Chem 2010;285: 4464-4471.

26 Mayr C, Hemann MT, Bartel DP. Disrupting the pairing between let-7 and Hmga2 enhances oncogenic transformation. Science 2007;315:1576-1579.

27 Wang $\mathrm{T}$, Zhang X, Obijuru L, et al. A micro-RNA signature associated with race, tumor size, and target gene activity in human uterine leiomyomas. Genes Chromosomes Cancer 2007;46:336-347.

28 Weidinger C, Krause K, Klagge A, et al. Forkhead box-O transcription factor: critical conductors of cancer's fate. Endocr Relat Cancer 2008;15:917-929.

29 Fei M, Zhao Y, Wang Y, et al. Low expression of Foxo3a is associated with poor prognosis in ovarian cancer patients. Cancer Invest 2009;27:52-59.

30 Lee YG, Macoska JA, Korenchuk S, et al. MIM, a potential metastasis suppressor gene in bladder cancer. Neoplasia 2002;4:291-294.

31 Parr C, Jiang WG. Metastasis suppressor 1 (MTSS1) demonstrates prognostic value and anti-metastatic properties in breast cancer. Eur J Cancer 2009;45: 1673-1683. 